\title{
The rat lung-worm Angiostrongylus cantonensis: A first report in South Africa
}

\author{
C E Archer, C C Appleton, S Mukaratirwa, K J Hope
}

To the Editor: A study of the parasites of invasive rats in the eThekwini Municipality of KwaZulu-Natal has led to this first report of Angiostrongylus (Parastrongylus) cantonensis, commonly known as the rat lung worm, in South Africa. A. cantonensis is clearly endemic in this region and probably also in other areas of South Africa. There are a few reports of this nematode from Africa (excluding South Africa): in rats and snails in Egypt (Fouad and Abdulla, 1978), in snails in Nigeria (Sowemimo and Asaolur, 2004), and a human case from Ivory Coast (1980). ${ }^{1}$ As humans are accidental hosts, the parasite cannot complete its life cycle, and immature worms lodge in the central nervous system where they elicit a condition known as eosinophilic meningitis.

\section{Background}

A. cantonensis is endemic in rats in Asia, the Pacific Islands, China, Australia and parts of North and South America, where human cases of eosinophilic meningitis are common. ${ }^{2}$

The life cycle of $A$. cantonensis involves the rat as definitive host and a mollusc (snail or slug) as intermediate host. Adult worms live in the pulmonary arteries of infected rats, where they mate and lay eggs. First-stage larvae hatch, ascend the bronchial tree, are swallowed, pass out in the rat faeces and are ingested by an intermediate host. Fish, shellfish, frogs and monitor lizards serve as paratenic hosts, while vegetables contaminated by slime-trails and faeces of molluscs are also sources of infection for the definitive host. If a rat ingests any of these, the infective third-stage larvae penetrate the gut wall and are carried via the bloodstream to the brain where they moult to fourthstage larvae, grow rapidly to about $1 \mathrm{~mm}$, re-enter the bloodstream and are carried to their final site in the pulmonary arteries, where they feed on blood and mature to adulthood. Mature females are 18.5 $33 \mathrm{~mm}$ in length and $0.28-0.5 \mathrm{~mm}$ in breadth, and males measure $15.5-22 \mathrm{~mm}$ by $0.25-0.35 \mathrm{~mm}$. One female may lay up to 15000 eggs per day. ${ }^{3}$

Humans are accidentally infected when they ingest raw or undercooked, infected intermediate or paratenic hosts, or vegetables contaminated with third-stage larvae. The parasites migrate from the gastrointestinal tract via the circulatory system, to the brain where they remain and die, so not completing their life cycle. There have been reports of patent infections (i.e. adolescent worms reach the lungs, mature to adults, mate and lay eggs) where the patients have died, but these are exceptional cases. ${ }^{4}$

Many species of snails and slugs serve as intermediate hosts for $A$. cantonensis but no survey has been done in South Africa.

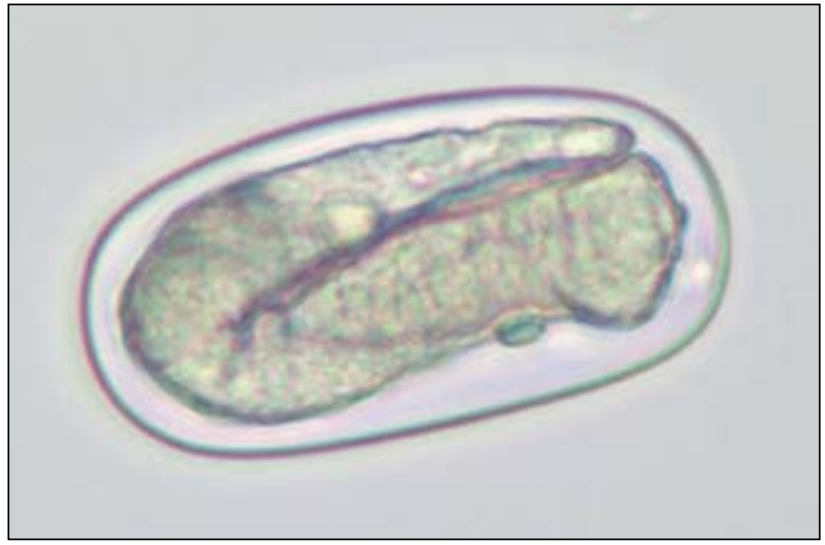

Fig. 1. A. cantonensis egg.

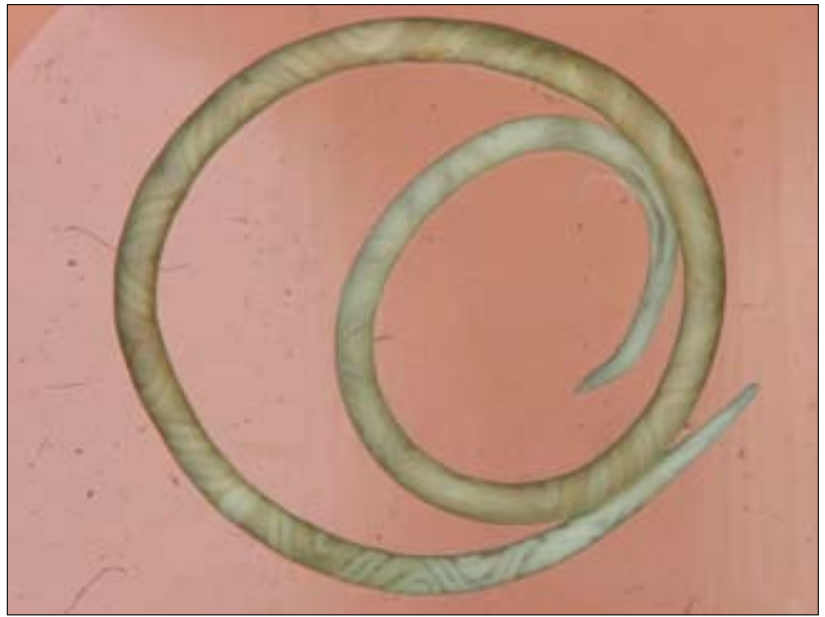

Fig. 2. A. cantonensis female.

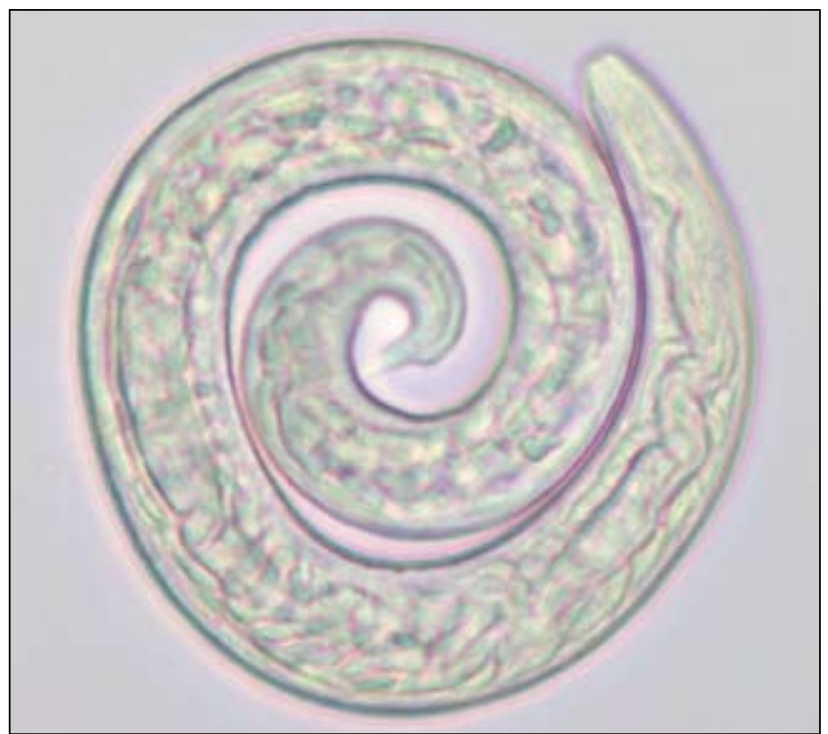

Fig. 3. A. cantonensis larva. 


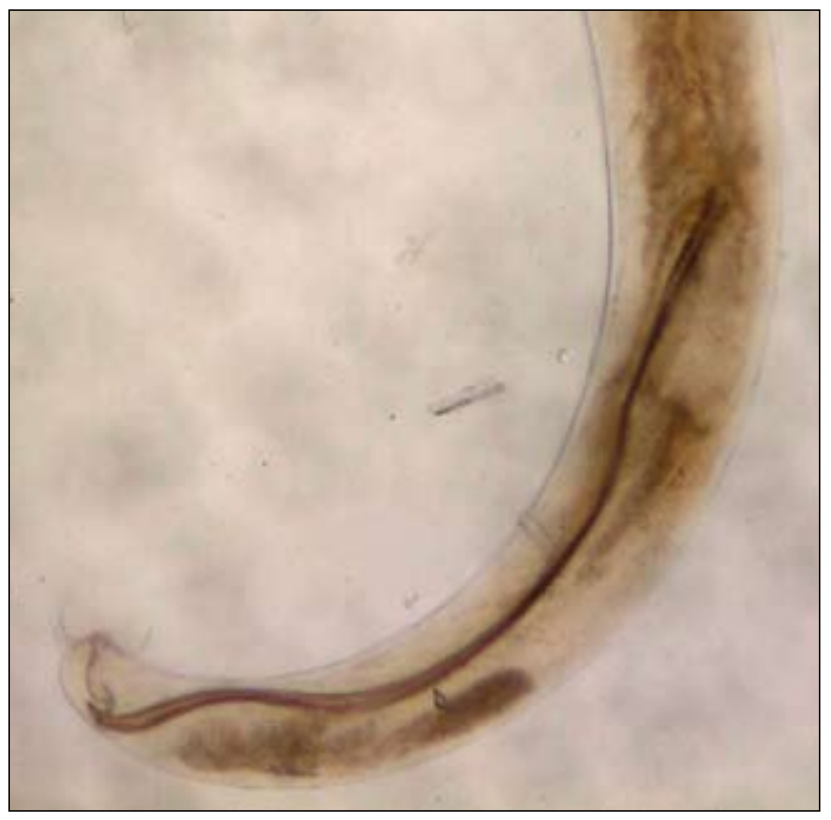

Fig. 4. A. cantonensis male rear end.

\section{The study}

eThekwini Health's Vector Control Programme has authorisation from the Durban Natural Science Museum (permit no. 4827/2007) to collect live specimens of Rattus spp. in baited traps. Our study in 2009 on parasites of invasive rats fitted in with this programme (approval by University of KwaZulu-Natal's Ethics Committee: Ref. 031/09/Animal). A total of 398 Rattus spp. (391 R. norvegicus and 7 R. rattus) were trapped during the year. Once killed, all ecto- and endoparasites were recovered for identification. Detailed results for these will be published in other journals. The heart and lungs were examined under a dissecting microscope.

Adult worms were isolated from the heart, pulmonary arteries and arterioles of 56 rats, ( 55 R. norvegicus and $1 R$. rattus), giving an overall prevalence of $14.1 \%$. Polymerase chain reaction (PCR) testing confirmed the identification of the worms as A. cantonensis. Further PCR testing and parasite morphology will be carried out and the results published.

\section{Clinical information}

When healthy patients become infected with $A$. cantonensis, the larvae and sub-adults remain in the CNS, commonly causing eosinophilic meningitis/meningoencephalitis. Other symptoms may result from human infections with this parasite and, although not as common, may cause severe illness and occasionally death.

After being ingested, infective larvae migrate to the CNS, resulting in eosinophilia in the CSF (30 - 45\%; 16 - 72\%) and to a lesser degree in the peripheral blood (20 - 32\%; $0-19 \%){ }^{5,6}$ (The first pair of figures in each set of parentheses (i.e. $30-45 \%$ and $20-32 \%$ ) were taken from Panackel et al., and the second set from Koo et al.) Clinical symptoms may also begin at this time or up to 4 weeks later. Patients most commonly present with severe headaches, neck stiffness, nausea, vomiting, confusion and hyperaesthesia. Other symptoms include papilloedema, hemiparesis, facial paralysis, hyperreflexia, fever and visual problems.?
Human angiostrongyliasis is usually mild and self-limiting, and recovery takes place within a week, but paraesthesiae and muscular weakness may persist for years and represent chronic forms of the disease. Such cases may indicate heavy infections.

A spinal tap is necessary for constructing a diagnosis of angiostrongyliasis, as eosinophilia is initially more likely to be seen in the CSF than in peripheral blood, and A. cantonensis larvae may occasionally be identified. ${ }^{8}$ It is advisable to specifically request the laboratory to perform an eosinophil count on the CSF. This requires centrifuging of the sample, making a smear from the deposit and staining with a routine haematological stain, such as Wright's.

There is no specific treatment, but high doses of the corticosteroid, prednisone (40 - $60 \mathrm{mg}$ daily) for a few weeks, or in chronic cases a few months, is helpful. ${ }^{9}$ CSF aspiration is effective for the relief of intracranial pressure and consequently the persistent headaches experienced by most patients. Other therapies include analgesics, antibiotics and anthelminthics. ${ }^{7}$

Although angiostrongyliasis is the most common cause of eosinophilic meningitis, the differential diagnoses include other infectious diseases, allergic reactions and malignancies. Among the parasitic diseases included here is gnathostomiasis - caused by the Asian nematode Gnathostoma spinigerum. ${ }^{5}$

\section{Conclusions}

A. cantonensis is clearly endemic in the urban rat population of the eThekwini area of KwaZulu-Natal, and may be more widespread. It might also have spread to the indigenous rodent community. Since $A$. cantonensis is the most common cause of eosinophilic meningitis in people, a retrospective analysis of all cases of eosinophilic meningitis of unknown aetiology is necessary to assess the likelihood of human A. cantonensis infections occurring in South Africa.

Although fatalities are uncommon in eosinophilic meningitis caused by angiostrongyliasis, young children and immunocompromised people are at risk of developing a fatal patent infection (i.e. the larvae migrate to the heart and lungs, mate and lay eggs) with severe lung pathology. This is consequently another opportunistic infection to be aware of in AIDS patients!

\section{References}

1. Nozais J-P, Moreau J, Morlier G, Kouame J, Doucet J. Premier cas de méningite a éosinophiles en Côte-d'Ivoire avec présence d'un Parastrongylus sp. dans le liquid céphalorachidien. Bull Soc Pathol Côte-d'Ivoire avec présence

2. Foronda P, López-González M, Miquel J, et al. Finding of Parastrongylus cantonensis (Chen, 1935) in Foronda P, López-González M, Miquel J, et al. Finding of Parastrongylus can
Rattus rattus in Tenerife, Canary Islands (Spain). Acta Trop 2010;114:123-127.

3. Mackerras MJ, Sandars DF. The life history of the rat lung-worm, Angiostrongylus cantonensis (Chen) (Nematoda: Metastrongylidae). Austr J Zoo 1955;3:1-21.

4. Pirisi M, Gutiérrez Y, Minini C, et al. Fatal human pulmonary infection caused by an Angiostrongyluslike nematode. Clin Infect Dis 1995;20:59-65.

5. Panackel C, Vishad, Cheriuan G, Vijayakumar, Sharma RN. Eosinophilic meningitis due to Angiostrongylus cantonensis. Indian j Med Micro 2006;24(3):220-221.

6. Koo J, Pien F, Kliks MM. Angiostrongylus (Parastrongylus) eosinophilic meningitis. Rev Infect Dis 1988;10(6):1155-1162.

7. Punyagupta S, Juttijudata P, Bunnag T. Eosinophilic meningitis in Thailand: Clinical studies of 484 typical cases probably caused by Angiostrongylus cantonensis. Am J Trop Med Hyg 1975;24:921-931.

8. Kuberski T, Bart RD, Briley JM, Rosen L. Recovery of Angiostrongylus cantonensis from cerebrospinal Kuberski T, Bart RD, Briley JM, Rosen L. Recovery of Angiostrongylus can
fluid of a child with eosinophilic meningitis. J Clin Micro 1979;9:629-631.

9. Pien FD, Pien BC. Angiostrongylus cantonensis Eosinophilic meningitis. Int J Infect Dis 1999;3(3):161Pien $\mathrm{F}, \mathrm{P}$.
163.

Accepted 4 October 2010 